\title{
Algorithm of Your Identification Quantities \\ on the Distance and Speed of Interference \\ in the Doppler Radar in the Interests of Forming \\ the Reliable Estimates of the Distance and Speed \\ at the Adaptation of the Air Objective
}

\author{
Alexander V. Bogdanov, \\ Denis V. Zakomoldin, Jaroslav S. Dokuchaev, \\ Viktor A. Novichenok and Igor V. Kochetov* \\ Military Academy of Aero-Space Defense \\ named after the Marshal of Soviet Union G.K. Zhukov \\ 50 Zhigareva Str., Tver, 170022, Russia
}

Received 14.12.2017, received in revised form 21.06.2018, accepted 29.11.2018

In this article, an algorithm has been developed that allows idle Doppler radar stations to influence the distance and speed of interference in various combination of them and, depending on the results, to form reliable estimates of range and speed using only the true values corresponding to the formation their motion parameters, excluding distortion-distorted values from the processing. Also, in this paper we present the results of modeling aimed at estimating the probabilistic and accuracy characteristic of a developed algorithm.

Keywords: interference, Doppler radar.

Citation: Bogdanov A.V., Zakomoldin D.V., Dokuchaev Ja.S., Novichenok V.A., Kochetov I.V. Algorithm of your identification quantities on the distance and speed of interference in the doppler radar in the interests of forming the reliable estimates of the distance and speed at the adaptation of the air objective, J. Sib. Fed. Univ. Eng. technol., 2019, 12(1), 30-40. DOI: $10.17516 / 1999-494 X-0103$.

(c) Siberian Federal University. All rights reserved

This work is licensed under a Creative Commons Attribution-NonCommercial 4.0 International License (CC BY-NC 4.0).

* Corresponding author E-mail address: kuchin.a.a@gmail.com, denjuga68@yandex.ru 


\title{
Алгоритм идентификации уводящих по дальности \\ и скорости помех в импульсно-доплеровских РЛС \\ В интересах формирования
}

достоверных оценок дальности и скорости

при сопровождении воздушной цели

\author{
А.В. Богданов, Д.В. Закомолдин, \\ Я.С. Докучаев, В.А. Новичёнок, И.В. Кочетов \\ Военная академия воздушно-космической оборонь \\ им. Маршала Советского Союза Г.К. Жукова \\ Россия, 170022, Тверь, ул. Жигарева, 50
}

В статье разработан алгоритм, позволяющий идентифицировать в импульсно-доплеровских радиолокаџионных станциях воздействие уводящих по дальности и скорости помех в различных их сочетаниях и в зависимости от результатов идентификации формировать достоверные оценки дальности и скорости, используя только истинные значения соответствуюших параметров движения, исключая из обработки искаженные помехой значения. Приведень результаты моделирования, направленные на оценку вероятностных и точностных характеристик разработанного алгоритма.

Ключевые слова: помехи, импульсно-доплеровская РЛС.

\section{Введение}

Анализ современного парка вооружения и военной техники наземного базирования, в частности образцов зенитных ракетных систем и комплексов, а также среднесрочной перспективы их развития, показал, что значительное количество РЛС наземного базирования функционирует по импульсно-доплеровскому (ИД) принципу обработки сигналов, в которых основным информативным параметром является доплеровская частота. Это, прежде всего, обусловлено такими достоинствами ИД РЛС, как [1]:

- возможность обеспечения большей по сравнению с другими типами РЛС дальности обнаружения целей за счет когерентного накопления энергии отраженных сигналов в узкополосных доплеровских фильтрах;

- возможность подавлять мешающие отражения от поверхности земли, а также преднамеренные пассивные помехи, выделяя при этом сигналы движущихся целей;

- возможность выполнять разделение (разрешение) целей по скорости сближения с целью и оценивать ее с высокой точностью;

- возможность обеспечивать высокую разрешающую способность по угловым координатам при наблюдении групповых целей за счет разности радиальных скоростей целей;

- возможность выполнять распознавание целей по особенностям структуры доплеровских спектров отраженных сигналов, которые к настоящему времени еще не все реализованы.

Кроме того, известно о все нарастающей тенденции применения средств радиоэлектронного подавления в современных вооруженных конфликтах [2-4], поэтому вопросам обеспече- 
ния помехоустойчивой работы ИД РЛС целесообразно уделять значительное внимание еще на этапе разработки и проектирования.

С учетом того, что, во-первых, одной из наиболее опасных помех для ИД РЛС является помеха, искажающая доплеровскую частоту, а именно уводящая по скорости (доплеровской частоте) (УС) помеха, наибольший эффект от воздействия которой достигается при дополнительной организации уводящей по дальности (УД) помехи, а во-вторых, что конечной задачей РЛС при сопровождении ВЦ служит формирование достоверных оценок параметров ее движения, целью статьи является разработка алгоритма идентификации уводящих по дальности и скорости помех в ИД РЛС в интересах формирования достоверных оценок дальности и скорости при сопровождении воздушных целей, а также оценка вероятностных и точностных характеристик разработанного алгоритма.

\section{Алгоритм идентификации}

На рис. 1 приведена блок-схема алгоритма, состоящего из блока быстрого преобразования Фурье (БПФ), формирователя наблюдения, анализатора, трех оптимальных фильтров сопровождения (ОФ $\Phi_{\mathrm{vд}}, \mathrm{O} \Phi_{\mathrm{v}}$, OФ $\left.\Phi_{\mathrm{vK}}\right)$, основанных на алгоритме калмановской фильтрации, интегратора, вычислителя и формирователя оценок.

Функционирует данный алгоритм следующим образом.

На вход блока БПФ на промежуточной частоте с выхода приемника РЛС поступает сигнал $\mathrm{S}(\mathrm{t})$, отраженный от ВЦ, который подвергается узкополосной доплеровской фильтрации на основе процедуры БПФ и преобразуется в амплитудно-частотный спектр, составляющие которого обусловлены отражениями сигнала от планера сопровождаемой ВЦ и вращающихся частей КНД ее силовой установки.

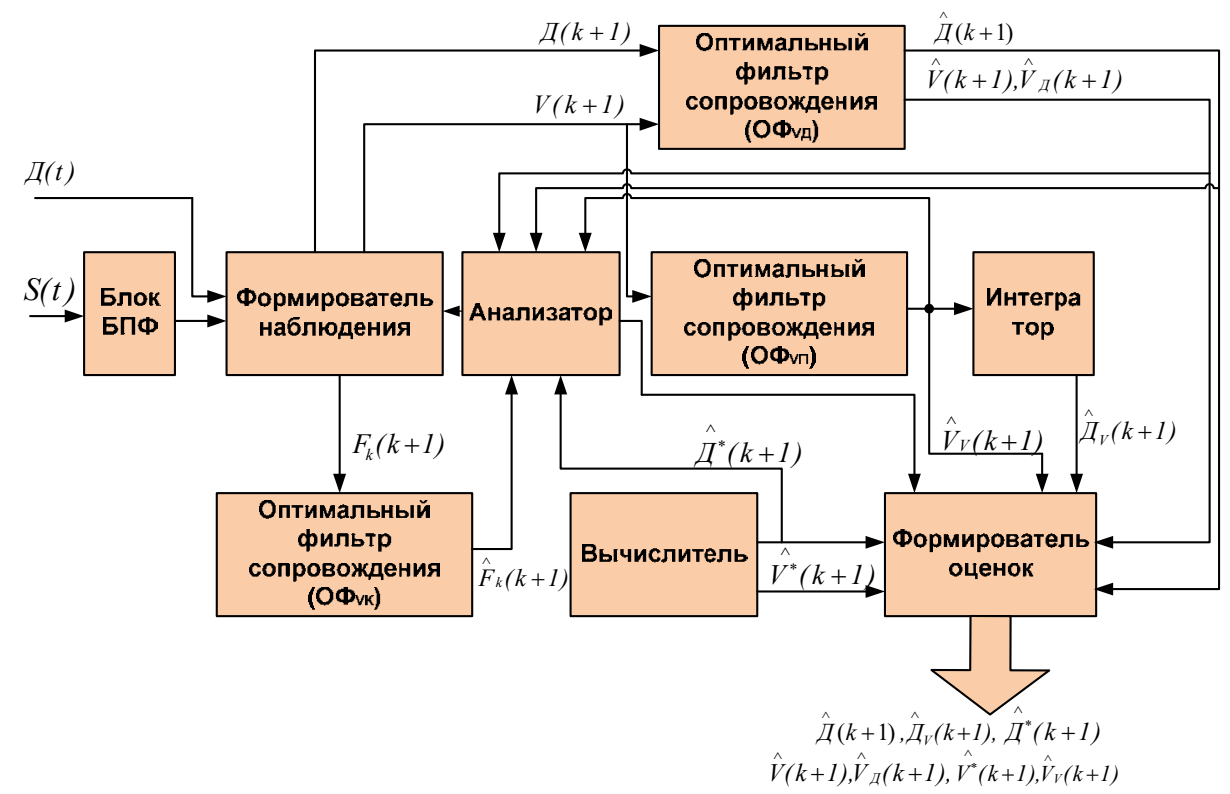

Рис. 1. Блок-схема алгоритма идентификации уводящих помех

Fig. 1. Block diagram of the identification of the escaping noise

$$
-32-
$$


В формирователе наблюдения, во-первых, определяется отсчет доплеровской частоты $F_{n}(k+1)$, соответствующий максимальной амплитуде спектральной составляющей спектра сигнала, который соответствует его отражениям от планера ВЦ, во-вторых, данный отсчет доплеровской частоты $F_{n}(k+1)$ преобразуется в значение скорости как $V(k+1)=\lambda F_{n}(k+1) / 2$ (где $\lambda$ - рабочая длина волны РЛС), в-третьих, поступающее на вход измерение дальности в непрерывном времени Д( $(t)$ преобразуется в дискретные отсчеты дальности Д( $k+1)$, в-четвертых, определяется отсчет доплеровской частоты $F_{k}(k+1)$, соответствующий максимальной амплитуде спектральной составляющей спектра сигнала, находящейся справа по доплеровской частоте относительно спектральной составляющей сигнала, отраженного от планера ВЦ. В результате на выходах формирователя наблюдения формируются наблюдения (измерения) значений Д( $k+1), V(k+1)$ и $F_{k}(k+1)$. Причем дискретные измерения Д( $\left.k+1\right)$ и $V(k+1)$ поступают на соответствующие входы оптимального фильтра сопровождения $\left(О \Phi_{\mathrm{v}, д}\right)$, работающего в соответствии с процедурой многомерной линейной дискретной калмановской фильтрации

$$
\begin{aligned}
& P^{-}(k+1)=\Phi(k) P(k) \Phi^{T}(k)+Q(k+1) ; \\
& \Psi(k+1)=H(k+1) P^{-}(k+1) H^{T}(k+1)+R(k+1) ; \\
& S(k+1)=P(k+1) H^{T}(k+1) \Psi^{1}(k+1) ; \\
& Z(k+1)=Y(k+1)-H(k+1) \hat{X}(k+1) ; \\
& \hat{X}(k+1)=\Phi(k) \hat{X}(k)+S(k+1) Z(k+1) ; \\
& P(k+1)=[I-S(k+1) H(k+1)] P(k+1),
\end{aligned}
$$

где

$k=0,1, \ldots, \mathrm{K}, \ldots,-$ номер такта работы фильтра;

$P^{-}(k+1)$ и $V(k+1)$ - ковариационные матрицы ошибок экстраполяции и фильтрации соответственно;

$\Phi(k)$ - переходная матрица состояния;

$Q(k+1)$ и $R(k+1)$ - ковариационные матрицы шумов возбуждения и наблюдения соответственно;

$S(k+1)$ - матрица весовых коэффициентов;

$I_{\wedge}$ - единичная матрица;

$X(k)$ и $X(k+1)$ - вектор текущих и экстраполированных оценок радиальных функционально-связанных координат взаимного перемещения носителя РЛС и воздушной цели;

$H(k+1)$ - матрица наблюдения;

$Y(k+1)$ - вектор наблюдения;

$Z(k+1)$ - матрица невязок измерения;

$\Psi(k+1)$ - матрица априорных ошибок фильтрации;

«-1»- операция вычисления обратной матрицы;

«т»- операция транспонирования матрицы,

априорные сведения в котором представлены в виде динамической модели 


$$
\begin{aligned}
& \dot{\not}(t)=V_{0}(t)+\Delta V(t), \ldots \ldots . Д(0)=Д_{0} \\
& \dot{\Delta V}(t)=a(t), \ldots \ldots \ldots . \Delta V(0)=\Delta V_{0} \\
& \dot{a}(t)=-\alpha a(t)-\beta \Delta V(t)+\sqrt{2 \alpha \sigma^{2}} n(t), \ldots \ldots . . a(0)=a_{0}, \\
& \dot{V}_{0}(t)=0, \ldots . . V_{0}(0)=V_{00}
\end{aligned}
$$

где

$Д(t), V(t)=V_{0}(t)+\Delta V(t)$ и $a(t)-$ радиальные функционально-связанные координаты: соответственно дальность, скорость и ускорение сближения РЛС с воздушной целью;

$V_{0}(t)$ и $\Delta V(t)$ - соответственно детерминированная и флюктуационная составляющие скорости сближения носителя РЛС с воздушной целью;

$\alpha$ - величина, обратная времени корреляции скоростных флюктуаций взаимного перемещения носителя РЛС и воздушной цели;

$\beta$ - квадрат собственной частоты скоростных флюктуаций взаимного перемещения носителя РЛС и воздушной цели;

$\sigma$ - среднеквадратическое отклонение флюктуаций ускорения взаимного перемещения носителя РЛС и воздушной цели;

$n(t)$ - формирующий белый гауссовский шум с нулевым математическим ожиданием и единичной интенсивностью, представляемой в процедуре (1) - (6) матрицами $\Phi(k+1)$ и $Q(k+1)$, размерностями $4 \times 4$, ненулевыми элементами которых являются соответственно $\phi_{1,1}=\phi_{2,1}=\phi_{4,4}=1$; $\phi_{1,2}=\phi_{1,4}=\phi_{2,3}=\mathrm{T} ; \phi_{3,2}=-\beta \mathrm{T} ; \phi_{3,3}=1-\alpha \mathrm{T} ;$ и $\mathrm{q}_{3,3}=2 \alpha \mathrm{T} \sigma^{2}$, где Т - период дискретизации.

С выхода оптимального фильтра сопровождения $\left(О \Phi_{\mathrm{v}, Д}\right)$ оценки дальности Д( $\left.k+1\right)$ и скорости $\hat{V}(k+1)$ поступают на соответствующие входы формирователя оценок и анализатора. Кроме того, дискретные отсчеты скорости $V(k+1)$ поступают на вход оптимального фильтра сопровождения планерной составляющей $\left(О \Phi_{\mathrm{vп}}\right)$, работающего также в соответствии с процедурой многомерной линейной дискретной калмановской фильтрации (1) - (6), априорные сведения в котором даны в виде динамической модели

$$
\begin{aligned}
& \Delta \dot{V}(t)=a(t), \ldots \ldots \ldots . \Delta V(0)=\Delta V_{0} \\
& \dot{a}(t)=-\alpha a(t)-\beta \Delta V(t)+\sqrt{2 \alpha \sigma^{2}} n(t), \ldots \ldots . . a(0)=a_{0}, \\
& \dot{V}_{0}(t)=0, \ldots . V_{0}(0)=V_{00}
\end{aligned}
$$

представляемой в процедуре (1) - (6) матрицами $\Phi(k)$ и $Q(k+1)$, размерностями $3 \times 3$, ненулевыми элементами которых являются соответственно $\phi_{1,1}=\phi_{3,3}=1 ; \phi_{1,2}=\mathrm{T} ; \phi_{2,1}=-\beta \mathrm{T} ; \phi_{2,2}=1-\alpha \mathrm{T}$ и $\mathrm{q}_{2,2}=$ $2 \alpha \sigma^{2}$, с выхода которого оценка скорости $\hat{V}_{V}(k+1)$ также поступает на соответствующие входы формирователя оценок и анализатора, а ее интегрированное значение с выхода интегратора в виде оценки дальности $\hat{Д}_{V}(k+1)$ также поступает на соответствующий вход формирователя оценок.

Одновременно отсчеты доплеровских частот $F_{k}(k+1)$ с выхода формирователя наблюдения поступают на вход оптимального фильтра сопровождения $\left(О \Phi_{\mathrm{VK}}\right)$ первой компрессорной составляющей спектра сигнала, работающего аналогично оптимальному фильтру сопровождения $\left(О \Phi_{\mathrm{V}}\right)$ в соответствии с процедурой многомерной линейной дискретной калмановской 
фильтрации (1) - (6) и динамической моделью, аналогичной выражениям (8), за исключением того, что радиальные функционально-связанные флюктуационные составляющие модели и ее параметры соответствуют взаимному перемещению не планера ВЦ и РЛС, а лопаток рабочего колеса первой ступени КНД силовой установки ВЦ и РЛС. Кроме того, величина $\mathrm{V}_{01}$ будет соответствовать постоянному значению доплеровской частоты (скорости), отличной от планерной составляющей доплеровской частоты (скорости) на величину разноса доплеровских частот (скоростей), обусловленных отражениями сигнала от планера ВЦ и первой ступени КНД ее силовой установки $\left(\mathrm{V}_{02}\right)$. Сформированная на выходе оптимального фильтра сопровождения $\left(О \Phi_{\mathrm{v \kappa}}\right)$ оценка $\hat{F}_{\kappa}(k+1)$ поступает на соответствующий вход анализатора.

В вычислителе на основе динамической модели радиальных функционально-связанных координат (без учета измеренных значений дальности и скорости) вычисляются оценки дальности $\hat{\not}(k+1)$ и скорости $\hat{V}(k+1)$, которые подаются на соответствующие входы формирователя оценок, а оценка дальности Д $(k+1)$ дополнительно поступает на соответствующий вход анализатора.

. В анализаторе, во-первых, вычисляется модуль производной оценки разности $\left|\Delta F_{n \kappa}(k+1)\right|$ между оцененными значениями доплеровских частот, обусловленных отражениями от планера $\hat{F}_{n}(k+1)$ (преобразованное в анализаторе значение оценки скорости в доплеровскую частоту как $\left.\hat{F}_{n}(k+1)=2 \hat{V}(k+1) / \lambda\right)$ и лопаток рабочего колеса первой ступени $\hat{F}_{\kappa}(k+1)$ КНД силовой установки воздушной цели, которая сравнивается с пороговым значением $\varepsilon$, близким к нулю [5],

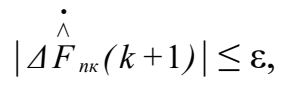

во-вторых, вычисляется модуль разности между оценкой производной дальности $\hat{Д}(k+1)$ и оценкой скорости $\hat{V}_{V}(k+1)$, величина которого сравнивается с порогом $\varepsilon_{1}$

$$
\left|\hat{\lambda}(k+1)-\hat{V}_{V}(k+1)\right| \leq \varepsilon_{1} ;
$$

в-третьих, вычисляется модуль разности между оценкой дальности $\hat{Д}(k+1)$ и вычисленной дальностью $\hat{\not}(k+1)$ на основе динамической модели радиальных функционально-связанных координат (7), величина которого сравнивается с порогом $\varepsilon_{2}$

$$
|\hat{\not}(k+1)-\hat{д}(k+1)| \leq \varepsilon_{2} .
$$

В анализаторе осуществляется анализ выполнения условий (9), (10) и (11). В результате анализа на его выходах формируются следующие команды.

При одновременном выполнении условий (9) и (10), что свидетельствует об отсутствии уводящих по скорости и дальности помех, на вход формирователя наблюдения с выхода анализатора поступает команда, в результате выполнения которой на его выходе формируется наблюдение вида $Y(k+1)=[Д(k+1), V(k+1)]^{\mathrm{T}}$; в этом случае оценки дальности Д( $\left.k+1\right)$ и скорости $\hat{V}(k+1)$ с выхода оптимального фильтра сопровождения $\left(О \Phi_{\mathrm{v}, Д}\right)$ через формирователь оценок по соответствующей команде с выхода анализатора поступают на выход канала сопровождения ВЦ в РЛС.

При одновременном выполнении условия (9) и невыполнении условия (10), что свидетельствует о воздействии только уводящей по дальности помехи, на вход формирователя 
наблюдения с выхода анализатора поступает команда, в результате выполнения которой на его выходе формируется наблюдение вида $Y(k+1)=[V(k+1)]$; в этом случае оценки скорости $\hat{V}_{V}(k+1)$ с выхода оптимального фильтра сопровождения $\left(О \Phi_{\mathrm{vп}}\right)$ и дальности $\hat{Д}_{V}(k+1)$, полученные путем интегрирования оценки скорости в интеграторе, через формирователь оценок по соответствующей команде с выхода анализатора поступают на выход канала сопровождения ВЦ в РЛС.

При одновременном невыполнении условия (9), выполнении условия (10), что свидетельствует о воздействии уводящих по дальности и скорости помех с функционально-связанным законом увода, на вход формирователя наблюдения с выхода анализатора поступает команда, в результате выполнения которой на его выходе будет отсутствовать какое-либо наблюдение, т.е. $Y(k+1)=0$; в этом случае оценки дальности $\hat{Д}(k+1)$ и скорости $V(k+1)$ с выхода вычислителя через формирователь оценок по соответствующей команде с выхода анализатора поступают на выход канала сопровождения ВЦ в РЛС.

Аналогично при одновременном невыполнении условий (9) и (10), что свидетельствует о воздействии уводящих по дальности и скорости помех без функционально-связанного закона увода, на вход формирователя наблюдения с выхода анализатора поступает команда, в результате выполнения которой на его выходе будет также отсутствовать какое-либо наблюдение, т.е. $Y(k+1)=0$, и оценки дальности $\not$ ( $(k+1)$ и скорости $V(k+1)$ с выхода вычислителя через формирователь оценок по соответствующей команде с выхода анализатора поступают на выход канала сопровождения ВЦ в РЛС.

При одновременном невыполнении условия (9), (10) и выполнении условия (11), что свидетельствует о воздействии только уводящей по скорости помехи, на вход формирователя наблюдения с выхода анализатора поступает команда, в результате выполнения которой на его выходе формируется наблюдение вида $Y(k+1)=[Д(k+1)]$; в этом случае оценки дальности $\hat{д}(k+1)$ и скорости $\stackrel{\wedge}{V}(k+1)$ с выхода оптимального фильтра сопровождения $\left(О \Phi_{\mathrm{v}, Д}\right)$ через формирователь оценок по соответствующей команде с выхода анализатора поступают на выход канала сопровождения ВЦ в РЛС.

\section{Вероятностные и точностные характеристики разработанного алгоритма}

Оценка вероятностных и точностных характеристик разработанного алгоритма произведена на основе моделирования.

При моделировании были приняты следующие исходные данные.

1. Рабочая длина волны РЛС $-\lambda=4 \times 10^{-2} \mathrm{M}$.

2. Для флюктуационных составляющих планерной (индекс «1» и компрессорной (индекс «2») доплеровских частот соответственно $\alpha_{1} \approx 0,2$ Гц, $\beta_{1} \approx 0,4$ рад $^{2} \mathrm{c}^{-2}, \sigma_{1} \approx 0,2 \mathrm{м} / \mathrm{c}$ и $\alpha_{1} \approx 0,2$ Гц, $\beta_{1}$ $\approx 0,8 \mathrm{paд}^{2} \mathrm{c}^{-2}, \sigma_{1} \approx 0,1 \mathrm{M} / \mathrm{c}$.

3. Постоянное значение радиальной скорости сближения ВЦ-постановщика помех с РЛС $-330 \mathrm{~m} / \mathrm{c}$.

4. Уводящая по скорости помеха изменялась по линейному закону со скоростью увода 600 Гц/с. (С данным значением скорости увода организуется уводящая по скорости помеха на практике [3]). 
5. Уводящая по дальности помеха изменялась по линейному закону со скоростью увода $12 \mathrm{м} / \mathrm{c}$ (для случая воздействия только уводящей по дальности помехи и при зависимом с уводящей по скорости законом увода), 20 м/с (при ситуации независимого закона увода).

6. Шаг моделирования $-\mathrm{T}_{\text {д }}=0,1$.

7. Время воздействия помех $-\mathrm{T}_{\text {п }}=10 \mathrm{c}$.

8. Разрешающая способность по доплеровской частоте (ширина полосы пропускания узкополосного доплеровского фильтра) - 10 Гц (время когерентного накопления $\left.\mathrm{T}_{\mathrm{Kн}}=100 \mathrm{Mc}\right)$

Результаты оценок вероятностей идентификации уводящих помех в различных их сочетаниях, вычисленных на основе моделирования, представлены в табл. 1, в которой принято: $\mathrm{P}$ - вероятность правильной идентификации, $\mathrm{P}_{\text {ли }}$ - вероятность ложной идентификации.

В интересах дальнейшего исследования точностных показателей формируемых оценок была оценена сходимость фильтра сопровождения ВЦ по дальности и скорости, функционирующего в соответствии с выражениями (1) - (6), посредством анализа графиков, полученных в результате моделирования, представленных для примера на рис. 1 и 2 при отношении сигнал/ шум 20 дБ, которые свидетельствуют о сходимости синтезированного фильтра сопровождения ввиду того, что усредненное значение реального среднеквадратического отклонения ошибок

Таблица 1. Оценки вероятностей идентификации уводящих помех

Table 1. Estimation of probabilities of identification of escaping noise

\begin{tabular}{|c|c|c|c|c|}
\hline \multirow{3}{*}{$\begin{array}{c}\text { Вероятность } \\
\text { идентификации }\end{array}$} & \multicolumn{4}{|c|}{ Распознаваемая помеховая обстановка } \\
\hline & \multirow[b]{2}{*}{$\begin{array}{c}\text { Уводящая по } \\
\text { скорости помеха }\end{array}$} & \multirow[b]{2}{*}{$\begin{array}{c}\text { Уводящая по } \\
\text { дальности помеха }\end{array}$} & \multicolumn{2}{|c|}{ Уводящая по дальности и скорости помеха } \\
\hline & & & $\begin{array}{l}\text { Зависимый } \\
\text { закон увода }\end{array}$ & $\begin{array}{c}\text { Независимый } \\
\text { закон увода }\end{array}$ \\
\hline Р/ Рли & $\sim 0,9 / 10^{-5}$ & $\sim 0,85 / 10^{-5}$ & $\sim 0,7 / 10^{-5}$ & $\sim 0,75 / 10^{-5}$ \\
\hline
\end{tabular}

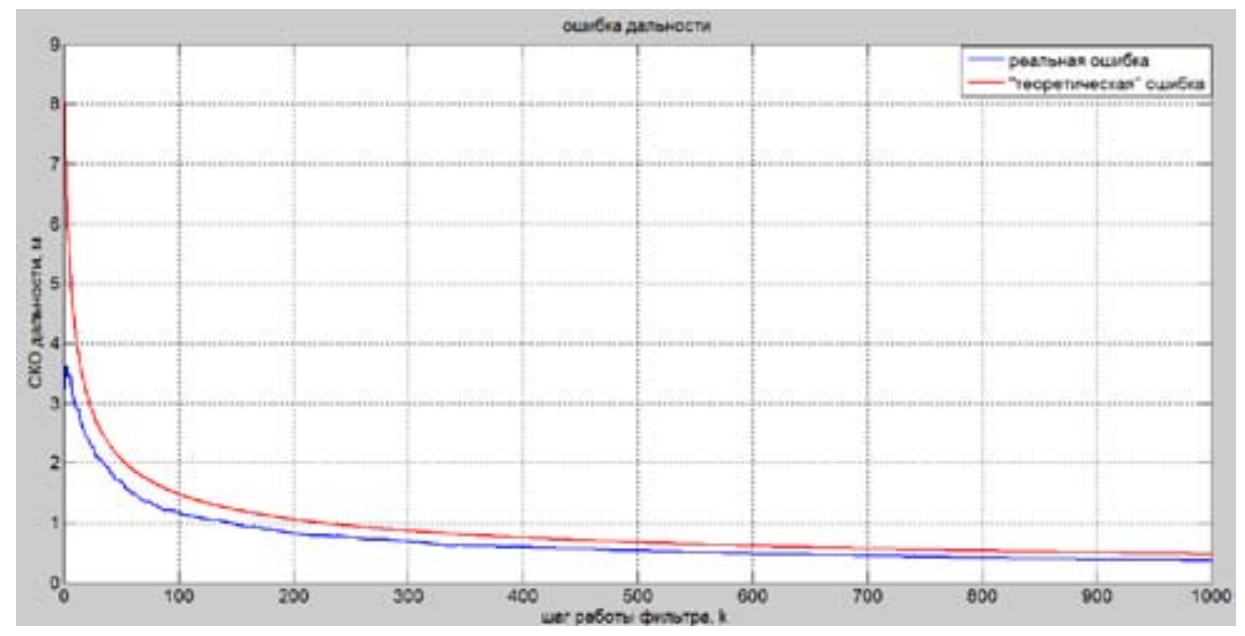

Рис. 2. Ошибки формирования оценок дальности. Отношение с $/ ш=20$ дБ

Fig. 2. Errors in the formation of range estimates. Signal-to-noise ratio $20 \mathrm{~dB}$ 


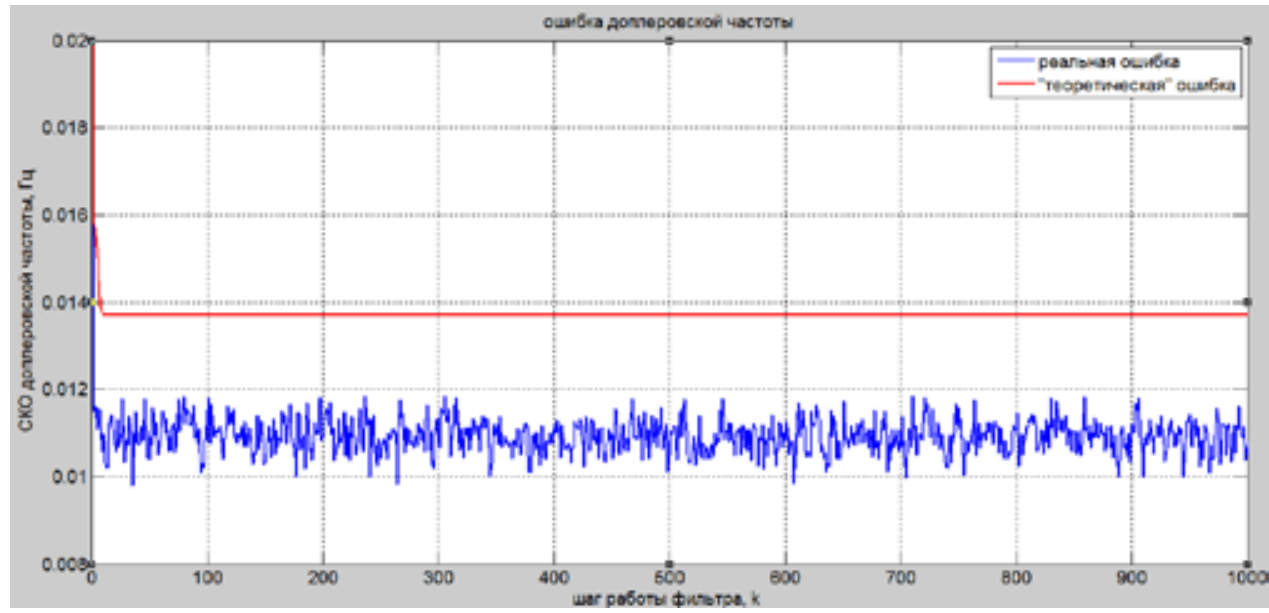

Рис. 3. Ошибки формирования оценок доплеровской частоты. Отношение с/ш = 20 дБ

Fig. 3. Errors in the formation of Doppler frequency estimates. Signal-to-noise ratio $20 \mathrm{~dB}$

фильтрации (синяя кривая) не превышает теоретический их уровень (красная кривая), рассчитанный в соответствии с выражением (6) [1].

При оценке точности формируемых оценок дальности и скорости были получены результаты, представленные в табл. 2-5 для различных ситуаций помеховой обстановки, которые ввиду того, что являются оценками, полученными на основе моделирования, были оценены доверительными оценками. В данных таблицах принято: q - отношение сигнал-шум; $\sigma_{\text {Д, }} \sigma_{\mathrm{F}}$ среднеквадратическое отклонение ошибок дальности и доплеровской частоты соответственно; $\sigma\left(\sigma_{д}\right)$ - среднеквадратическое отклонение оценки $\sigma_{д} ; \sigma\left(\sigma_{F}\right)$ - среднеквадратическое отклонение оценки $\sigma_{F} ; \mathrm{I}_{0,95}(Д), \mathrm{I}_{0,95}(\mathrm{~V})$ - доверительный интервал, который с доверительной вероятностью 0,95 накрывает оценки $\sigma_{д}$ и $\sigma_{\mathrm{F}}$ соответственно.

В табл. 2 представлены результаты для случая беспомеховой обстановки, когда оценки формируются с учетом наблюдения дальности и скорости.

В табл. 3 представлены результаты для случая воздействия уводящей по скорости помехи, когда оценки формируются с учетом наблюдения только дальности.

В табл. 4 представлены результаты для случая воздействия УД помехи, когда оценки формируются с учетом наблюдения только скорости.

В табл. 5 представлены результаты для случая воздействия УД и УС помех, когда оценки формируются без учета наблюдения.

Анализ табл. 2-5, отражающих результаты доверительного оценивания среднеквадратических ошибок формирования оценок дальности и доплеровских частот на выходах фильтров сопровождения, полученных на основе моделирования, показывает:

1) при увеличении отношения сигнал/шум для случая присутствия в качестве наблюдения на входе фильтра сопровождения хотя бы одной координаты $\sigma$ ошибок формируемых оценок дальности и доплеровских частот уменьшается;

2) оптимальными (наилучшими) из рассмотренных по критерию минимум $\sigma$ ошибок формируемых оценок дальности и доплеровских частот (фильтрации) являются оценки, 
Таблица 2. Результаты моделирования в беспомеховой обстановке

Table 2. Simulation results for the fussless situation

\begin{tabular}{|c|c|c|c|c|c|c|}
\hline \multicolumn{7}{|c|}{ В беспомеховой обстановке (на основе модели и наблюдения Д и V) } \\
\hline $\mathrm{q}$ & $\sigma Д$ & $\sigma(\sigma Д)$ & $\mathrm{I} 0,95(Д)$ & $\sigma \mathrm{F}$ & $\sigma(\sigma \mathrm{F})$ & $\mathrm{I} 0,95(\mathrm{~V})$ \\
\hline 14 & $\sim 1,3$ & 0,25 & $1 \ldots 1,6$ & $\sim 0,03$ & 0,005 & $0,01 \ldots 0,02$ \\
\hline 20 & $\sim 0,8$ & 0,15 & $0,5 \ldots 0,8$ & $\sim 0,01$ & 0,0008 & $0,01 \ldots 0,012$ \\
\hline
\end{tabular}

Таблица 3. Результаты моделирования для случая воздействия УС помехи

Table 3. Simulation results for the case of the effect of speed-escalating interference

\begin{tabular}{|c|c|c|c|c|c|c|}
\hline \multicolumn{6}{|c|}{ При воздействии только уводящей по скорости помехи (на основе модели и наблюдения Д) } \\
\hline $\mathrm{q}$ & $\sigma Д$ & $\sigma(\sigma Д)$ & $\mathrm{I} 0,95(Д)$ & $\sigma \mathrm{F}$ & $\sigma(\sigma \mathrm{F})$ & $\mathrm{I} 0,95(\mathrm{~V})$ \\
\hline 14 & $\sim 1,7$ & $\sim 0,3$ & $1,3 \ldots 2$ & 0,26 & 0,02 & $0,24 \ldots 0,28$ \\
\hline 20 & $\sim 1,2$ & $\sim 0,16$ & $1 \ldots 1,4$ & 0,25 & 0,015 & $0,24 \ldots 0,27$ \\
\hline
\end{tabular}

Таблица 4. Результаты моделирования для случая воздействия УД помехи

Table 4. Simulation results for the case of the effect of distance-leading interference

\begin{tabular}{|c|c|c|c|c|}
\hline \multicolumn{5}{|c|}{ При воздействии уводящей по дальности помехи (на основе модели и наблюдения $\mathrm{V})$} \\
\hline $\mathrm{q}$ & $\sigma Д$ & $\sigma \mathrm{F}$ & $\sigma(\sigma \mathrm{F})$ & $\mathrm{I} 0,95(\mathrm{~V})$ \\
\hline 14 & $\sim 3,4$ & 0,02 & 0,006 & $0,015 \ldots 0,025$ \\
\hline 20 & $\sim 3,4$ & 0,01 & 0,0008 & $0,01 \ldots 0,012$ \\
\hline
\end{tabular}

Таблица 5. Результаты моделирования для случая воздействия УД и УС помех

Table 5. Simulation results for the case of the effect of distance-leading and speed-escalating interference

\begin{tabular}{|c|c|c|c|c|c|}
\hline \multicolumn{6}{|c|}{$\begin{array}{c}\text { При одновременном воздействии уводящих по дальности и скорости помех } \\
\text { (на основе модели функционально связанных координат) }\end{array}$} \\
\hline$\sigma Д$ & $\sigma(\sigma Д)$ & $\mathrm{I} 0,95($ Д) & $\sigma \mathrm{F}$ & $\sigma(\sigma \mathrm{F})$ & $\mathrm{I}), 95(\mathrm{~V})$ \\
\hline 2,6 & 0,9 & $1,7 \ldots 3,4$ & 0,36 & 0,12 & $0,25 \ldots 0,43$ \\
\hline
\end{tabular}

сформированные на выходе фильтра сопровождения, на входе которого в качестве наблюдения присутствуют две функционально-связанные координаты (ситуация беспомеховой обстановки);

3) наихудшими из рассмотренных по критерию минимум б ошибок формируемых оценок дальности и доплеровских частот являются оценки, сформированные на выходе вычислителя, формирующего оценки без учета наблюдения на основе только динамической модели (ситуация одновременного воздействия уводящих по дальности и скорости помех);

4) в целом же при ширине полосы пропускания доплеровского фильтра порядка 10 Гц и длительности импульса порядка 1 мкс (разрешающая способность по дальности порядка 150 м) точностные характеристики оценок дальностей и доплеровских частот для всех рассма-

$$
-39-
$$


триваемых гипотез относительно помеховой обстановки находятся в пределах одного элемента разрешения, следовательно их нужно считать достаточными.

\section{Заключение}

Таким образом, применение предлагаемого алгоритма позволит, во-первых, идентифицировать присутствие или отсутствие совместного или раздельного воздействия уводящих по дальности и скорости помех, во-вторых, сформировать достоверные оценки радиальных функционально-связанных дальности до воздушной цели и скорости сближения ВЦ с РЛС при ее сопровождении с приемлемыми на практике характеристиками.

\section{Список литературы}

[1] Оиенивание дальности и скорости в радиолокаџионных системах. Ч.1. Под ред. А.И. Канащенкова и В.И. Меркулова. М.: Радиотехника, 2004.312 с. [Estimmation of distance and speed in radar systems P.1. Ed. A.I. Kanashchenkov and V.I. Merkulov. Moscow, Radio engineering, 2004, $312 \mathrm{p}$ (in Russian)]

[2] Радиоэлектронная борьба в Вооруженных силах Российской Федераиии. Тематический сборник 2012 - 2018 гг. [Electronic warfare in The armed forces of the Russian Federation. Thematic collection 2012-2018 (in Russian)]

[3] Защита радиолокационных систем от помех. Состояние и тенденции развития. Под ред. А.И. Канащенкова и В.И. Меркулова. М.: Радиотехника, 2003. 416 с. [Protection of Radar system from interference. Status and development trends. Ed. A.I. Kanashchenkov and V.I. Merkulov. Moscow, Radio engineering, 2003, 416 p (in Russian)]

[4] Метод повыщения помехозащищенности бортовой радиолокаџионной станции истребителя: монография / А.В. Богданов и др; рук. авт. коллектива Д.В. Закомолдин. Красноярск: Сиб. федер. ун-т, 2018. 182 с. [Method increases noise immunity onboard fighter's radar station. Monograth. A.V. Bogdanov, D.V. Zakomoldin and others. Krasnojarsk: Sib. Feder. Un-t, 2018, 182 p. (in Russian)]

[5] Способ сопровождения воздушной цели из класса «самолет с турбореактивным двигателем» при воздействии уводящей по скорости помехи. Д.В. Закомолдин, А.В. Богданов, И.А. Новиченок. Патент на изобретение № 2579353 МПК G01S13/52 (2006.01), Россия, заявка № 2015112501, приоритет 06.04.2015, зарегестрировано 04.03.2016 [Method track air targets from the class "plane turbojet engine" under the influence of the driving noise speeds. D.V. Zakomoldin, A.V. Bogdanov, I.A. Novichonok. Patent for invention No. 2579353, MPK G01S13/52 (2006.01), Russia, application No. 2015112501, priority 06.04.2015, registered 04.03.2016 (in Russian)] 